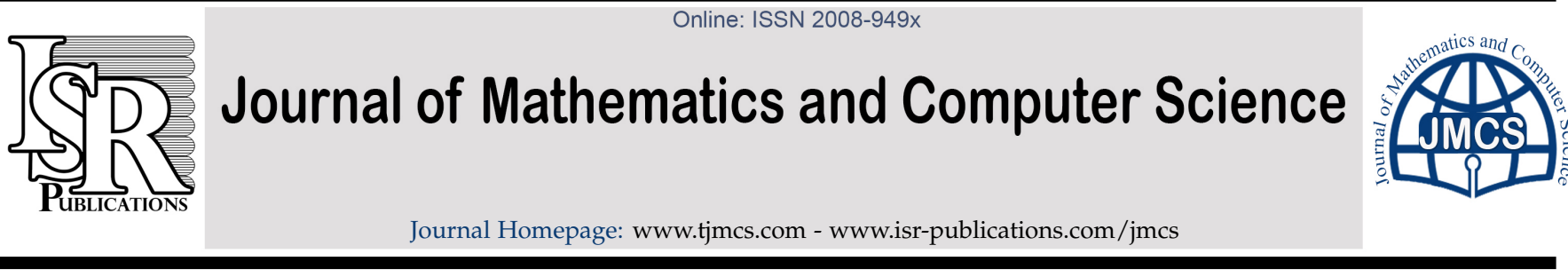

\title{
Another class of warped product CR-submanifolds in Kenmotsu manifolds
}

\author{
Siraj Uddin*, Azeb Alghanemi, Monia Fouad Naghi, Falleh Rijaullah Al-Solamy \\ Department of Mathematics, Faculty of Science, King Abdulaziz University, Jeddah 21589, Saudi Arabia.
}

\begin{abstract}
Recently, Arslan et al. [K. Arslan, R. Ezentas, I. Mihai, C. Murathan, J. Korean Math. Soc., 42 (2005), 1101-1110] studied contact CR-warped product submanifolds of the form $M_{T} \times_{f} M_{\perp}$ of a Kenmotsu manifold $\widetilde{M}$, where $M_{T}$ and $M_{\perp}$ are invariant and anti-invariant submanifolds of $\widetilde{M}$, respectively. In this paper, we study the warped product submanifolds by reversing these two factors, i.e., the warped products of the form $M_{\perp} \times_{f} M_{T}$ which have not been considered in earlier studies. On the existence of such warped products, a characterization is given. A sharp estimation for the squared norm of the second fundamental form is obtained, and in the statement of inequality, the equality case is considered. Finally, we provide two examples of non-trivial warped product submanifolds. (C)2017 All rights reserved.
\end{abstract}

Keywords: Warped products, contact CR-submanifolds, mixed totally geodesic, contact CR-warped products, Kenmotsu manifolds.

2010 MSC: 53C25, 53C40, 53C42, 53B25.

\section{Introduction}

To construct the examples of manifolds with negative curvature, the warped product manifolds were studied by Bishop and $\mathrm{O}^{\prime} \mathrm{Neill}$ in [4]. They defined these manifolds as follows: Let $M_{1}$ and $M_{2}$ be two Riemannian manifolds with Riemannian metrics $g_{1}$ and $g_{2}$, respectively, and a positive differentiable function $f$ on $M_{1}$. Consider a product manifold $M_{1} \times M_{2}$ with its projections $\pi_{1}: M_{1} \times M_{2} \rightarrow M_{1}$ and $\pi_{2}: M_{1} \times M_{2} \rightarrow M_{2}$. Then their warped product manifold $M=M_{1} \times_{f} M_{2}$ is the Riemannian manifold $M_{1} \times M_{2}=\left(M_{1} \times M_{2}, g\right)$ equipped with the Riemannian structure such that

$$
g(X, Y)=g_{1}\left(\pi_{1 \star} X, \pi_{1 \star} Y\right)+\left(f \circ \pi_{1}\right)^{2} g_{2}\left(\pi_{2 \star} X, \pi_{2 \star} Y\right),
$$

for any vector field $X, Y$ tangent to $M$, where $\star$ is the symbol for the tangent maps. A warped product manifold $M=M_{1} \times_{f} M_{2}$ is said to be trivial or simply a Riemannian product manifold, if the warping function $f$ is constant. Let $X$ be a vector field tangent to $M_{1}$ and $Z$ be an another vector field on $M_{2}$, then from [4, Lemma 7.3], we have

$$
\nabla_{X} Z=\nabla_{Z} X=(X \ln f) Z,
$$

\footnotetext{
*Corresponding author

Email addresses: siraj.ch@gmail.com (Siraj Uddin), aalghanemi@kau.edu.sa (Azeb Alghanemi), mnaghi@kau.edu.sa (Monia Fouad Naghi), falleh@hotmail.com (Falleh Rijaullah Al-Solamy)

doi:10.22436/jmcs.017.01.13
} 
where $\nabla$ is the Levi-Civita connection on $M$. If $M=M_{1} \times_{f} M_{2}$ be a warped product manifold then $M_{1}$ is a totally geodesic submanifold of $M$ and $M_{2}$ is a totally umbilical submanifold of $M[4,5]$.

In the beginning of this century, Chen introduced the notion of warped product CR-submanifolds of Kaehler manifolds [5]. After that, many researchers extended this idea for other structures on a Riemannian manifold (some of them are cited here $[8,14]$ ). For the survey on warped product submanifolds, we refer to [6, 7].

On the other hand, several results on the warped product submanifolds of Kenmotsu manifolds appeared in $[1-3,11-13,17,18]$. In this paper, we study some geometric properties of warped product submanifolds of the form $M_{\perp} \times_{f} M_{T}$, where $M_{T}$ and $M_{\perp}$ are invariant and anti-invariant submanifolds of a Kenmotsu manifold $\widetilde{M}$, respectively. A characterization is given on the existence of such type of warped products. Also, we establish a relationship between the squared norm of second fundamental form $\|\sigma\|^{2}$ and the warping function $f$. The equality case in the statements of the inequality is considered. Furthermore, we construct non-trivial examples of warped product contact CR-submanifolds.

\section{Preliminaries}

Tanno [15] has classified the connected almost contact metric manifolds into 3 classes whose automorphism groups have maximum dimensions:

(a) Homogeneous normal contact Riemannian manifolds with constant $\varphi$ holomorphic sectional curvature;

(b) global Riemannian product of a line or a circle and a Kaehlerian space form;

(c) warped product spaces $L \times_{f} F$, where $L$ is a line and $F$ a Kaehlerian manifold.

Kenmotsu [10] studied the class (c) and characterized it by tensor equations. Later such manifolds were called Kenmotsu manifolds.

A $(2 n+1)$-dimensional Riemannian manifold $(\widetilde{M}, g)$ is said to be a Kenmotsu manifold, if it admits an endomorphism $\varphi$ of its tangent bundle $T \widetilde{M}$, a vector field $\xi$ and a 1-form $\eta$ satisfying the following conditions:

$$
\begin{gathered}
\varphi^{2}=-I+\eta \otimes \xi, \quad \varphi \xi=0, \quad \eta \circ \varphi=0, \quad \eta(\xi)=1, \\
g(\varphi X, \varphi Y)=g(X, Y)-\eta(X) \eta(Y), \quad \eta(X)=g(X, \xi), \\
\left(\widetilde{\nabla}_{X} \varphi\right) Y=g(\varphi X, Y) \xi-\eta(Y) \varphi X, \quad \widetilde{\nabla}_{X} \xi=X-\eta(X) \xi,
\end{gathered}
$$

for any vector fields $X, Y$ on $\widetilde{M}$, where $\widetilde{\nabla}$ is the Riemannian connection with respect to $g$.

Let $M$ be a submanifold of an almost contact metric manifold $\widetilde{M}$ with induced metric $g$ and if $\nabla$ and $\nabla^{\perp}$ are the induced connections on the tangent bundle $T M$ and the normal bundle $T^{\perp} M$ of $M$, respectively, then the Gauss-Weingarten formulas are respectively given by

$$
\widetilde{\nabla}_{X} Y=\nabla_{X} Y+\sigma(X, Y), \quad \widetilde{\nabla}_{X} V=-A_{V} X+\nabla_{X}^{\perp} V,
$$

for any vector field $X, Y$ tangent to $M$ and $V$ normal to $M$, where $\sigma$ and $A_{V}$ are the second fundamental form and the shape operator (corresponding to the normal vector field $V$ ) respectively for the immersion of $M$ into $\widetilde{M}$. They are related as $g(\sigma(X, Y), V)=g\left(A_{V} X, Y\right)$ where $g$ denotes the Riemannian metric on $\widetilde{M}$ as well as the one induced on $M$.

Let $\widetilde{M}$ be a Kenmotsu manifold and $M$ an m-dimensional submanifold tangent to $\xi$. For any $X$ tangent to $M$, we put

$$
\varphi X=P X+F X,
$$


where PX (resp. FX) denotes the tangential (resp. normal) component of $\varphi$ X. Then $\mathrm{P}$ is an endomorphism of tangent bundle TM and $F$ is a normal bundle valued 1-form on TM.

We denote by $\mathrm{H}$, the mean curvature vector, i.e.,

$$
H(p)=\sum_{i=1}^{m} \sigma\left(e_{i}, e_{i}\right),
$$

where $\left\{e_{1}, \cdots, e_{m}\right\}$ is an orthonormal basis of the tangent space $T_{p} M$, for any $p \in M$.

Also, we set

$$
\sigma_{i j}^{r}=g\left(\sigma\left(e_{i}, e_{j}\right), e_{r}\right), i, j=1, \cdots, m, \quad r=m+1, \cdots, 2 n+1,
$$

and

$$
\|\sigma\|^{2}=\sum_{i, j=1}^{m} g\left(\sigma\left(e_{i}, e_{j}\right), \sigma\left(e_{i}, e_{j}\right)\right) .
$$

For a differentiable function $f$ on an $n$-dimensional manifold $M$, the gradient $\vec{\nabla} f$ of $f$ is defined as $g(\vec{\nabla} f, X)=X f$, for any $X$ tangent to $M$. As a consequence, we have

$$
\|\vec{\nabla} \mathbf{f}\|^{2}=\sum_{i=1}^{n}\left(e_{i}(f)\right)^{2},
$$

for an orthonormal frame $\left\{e_{1} \cdots, e_{n}\right\}$ on $M$.

By the analogy with submanifolds in a Kaehler manifold, different classes of submanifolds in a Kenmotsu manifold were considered.

A submanifold $M$ tangent to $\xi$ is said to be invariant (resp. anti-invariant), if $\varphi\left(T_{p} M\right) \subset T_{p} M$, for all $p \in M$ (resp. $\varphi\left(T_{p} M\right) \subset T_{p}^{\perp} M$, for all $\left.p \in M\right)$.

A submanifold $M$ tangent to $\xi$ is said to be a contact CR-submanifold, if there exists a pair of orthogonal distributions $\mathcal{D}: p \rightarrow \mathcal{D}_{p}$ and $\mathcal{D}^{\perp}: p \rightarrow \mathcal{D}_{\mathrm{p}}^{\perp}$, for all $\mathrm{p} \in \mathrm{M}$ such that

(i) $\mathrm{TM}=\mathcal{D} \oplus \mathcal{D}^{\perp} \oplus\langle\sim\rangle$, where $\langle\xi\rangle$ is a 1-dimensional distribution spanned by $\xi$;

(ii) $\mathcal{D}$ is invariant by $\varphi$, i.e., $\varphi \mathcal{D}=\mathcal{D}$;

(iii) $\mathcal{D}^{\perp}$ is anti-invariant by $\varphi$, i.e., $\varphi \mathcal{D}^{\perp} \subseteq T^{\perp} M$.

Invariant and anti-invariant submanifolds are the special cases of contact CR-submanifolds. If we denote the dimensions of the distributions $\mathcal{D}$ and $\mathcal{D}^{\perp}$ by $d_{1}$ and $d_{2}$ respectively, then $M$ is invariant (resp. antiinvariant), if $d_{2}=0$ (resp. $d_{1}=0$ ).

For the integrability of the distributions $\mathcal{D}$ and $\mathcal{D}^{\perp}$, we have the following results for later use.

Lemma 2.1 ([12]). The $\varphi$ anti-invariant distribution $\mathcal{D}^{\perp} \oplus\langle\xi\rangle$ on a contact CR-submanifold of a Kenmotsu manifold $\widetilde{M}$ is always integrable.

Also, we can prove the following result for the integrability of the distribution $\mathcal{D}$.

Lemma 2.2. Let $M$ be a contact $C R$-submanifold of a Kenmotsu manifold $\widetilde{M}$. Then the $\varphi$ invariant distribution $\mathcal{D}$ is integrable, if and only if

$$
g\left(\nabla_{Y} X, Z\right)=g(\sigma(X, \varphi Y), \varphi Z)-\eta(Z) g(X, Y),
$$

for any $X, Y \in \mathcal{D}$ and $Z \in \mathcal{D}^{\perp} \oplus\langle\xi\rangle$. 
Proof. For any $X, Y \in \mathcal{D}$ and $Z \in \mathcal{D}^{\perp} \oplus\langle\xi\rangle$, we have

$$
\begin{aligned}
g([X, Y], Z) & =g\left(\varphi \widetilde{\nabla}_{X} Y, \varphi Z\right)+\eta(Z) g\left(\widetilde{\nabla}_{X} Y, \xi\right)-g\left(\widetilde{\nabla}_{Y} X, Z\right) \\
& \left.=g\left(\widetilde{\nabla}_{X} \varphi Y, \varphi Z\right)-g\left(\left(\widetilde{\nabla}_{X} \varphi\right) Y, \varphi Z\right)-\eta(Z) g\left(Y, \widetilde{\nabla}_{X} \xi\right)\right)-g\left(\widetilde{\nabla}_{Y} X, Z\right) .
\end{aligned}
$$

Using (2.2) and (2.3), we get

$$
g([X, Y], Z)=g(\sigma(X, \varphi Y), \varphi Z)-\eta(Z) g(X, Y)-g\left(\widetilde{\nabla}_{Y} X, Z\right) .
$$

Thus the result follows from the above equation.

Lemma 2.3. On a contact $C R$-submanifold $M$ of a Kenmotsu manifold $\widetilde{M}$. The following statement holds

$$
g([X, Y], Z)=g(\sigma(X, \varphi Y), \varphi Z)-g(\sigma(\varphi X, Y), \varphi Z),
$$

for any $X, Y \in \mathcal{D}$ and $Z \in \mathcal{D}^{\perp} \oplus\langle\xi\rangle$.

Proof. For any $X, Y \in \mathcal{D}$ and $Z \in \mathcal{D}^{\perp} \oplus\langle\xi\rangle$, we have

$$
g\left(\nabla_{X} Y, Z\right)=g\left(\widetilde{\nabla}_{X} Y, Z\right)=g\left(\varphi \widetilde{\nabla}_{X} Y, \varphi Z\right)+\eta(Z) g\left(\widetilde{\nabla}_{X} Y, \xi\right) .
$$

Using the covariant derivative property of $\varphi$ and the Kenmotsu structure equation (2.2), we derive

$$
g\left(\nabla_{X} Y, Z\right)=g\left(\widetilde{\nabla}_{X} \varphi Y, \varphi Z\right)-\eta(Z) g\left(Y, \widetilde{\nabla}_{X} \xi\right) .
$$

Then from (2.3) we get

$$
g\left(\nabla_{X} Y, Z\right)=g(\sigma(X, \varphi Y), \varphi Z)-\eta(Z) g(X, Y) .
$$

Interchanging $X$ and $Y$ in (2.4), we obtain

$$
g\left(\nabla_{Y} X, Z\right)=g(\sigma(Y, \varphi X), \varphi Z)-\eta(Z) g(X, Y) .
$$

Then from (2.4) and (2.5), we get the desired result.

Now, we have the following consequence of the above lemma.

Corollary 2.4. On a contact $C R$-submanifold $M$ of a Kenmotsu manifold $\widetilde{M}$. The invariant distribution $\mathcal{D}$ is integrable, if and only if

$$
\text { either } \sigma(X, \varphi \mathrm{Y})=\sigma(\varphi X, Y) \text { or } \sigma(\varphi X, Y) \in v \text {, }
$$

for any $X, Y \in \mathcal{D}$.

\section{Contact CR-warped products}

In [1], Arslan et al. studied warped product contact CR-submanifolds of the form $M_{T} \times_{f} M_{\perp}$, called contact CR-warped products of a Kenmotsu manifold $\widetilde{M}$, where $M_{T}$ and $M_{\perp}$ are invariant and antiinvariant submanifolds of $\widetilde{M}$, respectively. They establish an inequality for such type of warped products. In this paper, we study another type of warped products by reversing two factors which have not been considered in [1]. First, we prove the following results for later use.

Lemma 3.1. Let $M=M_{\perp} \times{ }_{f} M_{T}$ be a warped product submanifold of a Kenmotsu manifold $\widetilde{M}$ such that $\xi \in$ $\mathrm{TM}_{\perp}$, then we have 
(i) $g(\sigma(X, Y), \varphi Z)=\{(Z \ln f)-\eta(Z)\} g(X, \varphi Y)$;

(ii) $\mathrm{g}(\sigma(X, Z), \varphi W)=\mathrm{g}(\sigma(X, W), \varphi Z)$;

(iii) $g\left(\sigma(Z, W), \varphi W^{\prime}\right)=g\left(\sigma\left(Z, W^{\prime}\right), \varphi W\right)$;

for any $\mathrm{X}, \mathrm{Y} \in \mathrm{T} \mathrm{M}_{\mathrm{T}}$ and $\mathrm{Z}, \mathrm{W}, \mathrm{W}^{\prime} \in \mathrm{TM}_{\perp}$.

Proof. From (2.3) and (2.2), we have

$$
g(\sigma(X, Y), \varphi Z)=g\left(\widetilde{\nabla}_{X} Y, \varphi Z\right)=g\left(\left(\widetilde{\nabla}_{X} \varphi\right) Y, Z\right)-g\left(\widetilde{\nabla}_{X} \varphi Y, Z\right)=\eta(Z) g(\varphi X, Y)+g\left(\varphi Y, \widetilde{\nabla}_{X} Z\right),
$$

for any $X, Y \in T M_{T}$ and $Z \in T M_{\perp}$. Then the first part follows from the above relation by using (1.1). Also the second and third parts of the lemma can be derived by using (2.2), (2.3) and the orthogonality of vector fields.

If we interchange $X$ by $\varphi X$ and $Y$ by $\varphi Y$ in the first part of Lemma 3.1, then we get the following relations

$$
\begin{aligned}
& g(\sigma(\varphi X, Y), \varphi Z)=\{(Z \ln f)-\eta(Z)\} g(X, Y), \\
& g(\sigma(X, \varphi Y), \varphi Z)=\{\eta(Z)-(Z \ln f)\} g(X, Y),
\end{aligned}
$$

and

$$
g(\sigma(\varphi X, \varphi Y), \varphi Z)=\{(Z \ln f)-\eta(Z)\} g(X, \varphi Y)
$$

Corollary 3.2. On a contact $C R$-warped product submanifold $M=M_{\perp} \times{ }_{f} M_{T}$ of a Kenmotsu manifold $\widetilde{M}$, we have

$$
g(\sigma(\varphi X, Y), \varphi Z)=-g(\sigma(X, \varphi Y), \varphi Z)
$$

and

$$
\mathrm{g}(\sigma(\varphi \mathrm{X}, \varphi \mathrm{Y}), \varphi \mathrm{Z})=\mathrm{g}(\sigma(\mathrm{X}, \mathrm{Y}), \varphi \mathrm{Z})
$$

for any $\mathrm{X}, \mathrm{Y} \in \mathrm{T} \mathrm{M}_{\mathrm{T}}$ and $\mathrm{Z} \in \mathrm{TM}_{\perp}$.

Proof. The first part follows from (3.1) and (3.2) and the second part follows from Lemma 3.1 (i) and (3.3).

Theorem 3.3. Let $M$ be a contact CR-submanifold of a Kenmotsu manifold $\widetilde{M}$ such that $\xi$ is orthogonal to the invariant distribution $\mathcal{D}$. Then $M$ is a locally warped product submanifold, if and only if

$$
A_{\varphi Z} X=-\{(Z \mu)-\eta(Z)\} \varphi X
$$

for any $X \in \mathcal{D}$ and $Z \in \mathcal{D}^{\perp} \oplus\langle\xi\rangle$ for some smooth function $\mu$ on $M$ such that $Y(\mu)=0$, for any $Y \in \mathcal{D}$.

Proof. If $M$ is a contact $C R$-warped product submanifold, then for any $X \in T M_{T}$ and $Z, W \in T M_{\perp}$, we have

$$
g\left(A_{\varphi Z} X, W\right)=g(\sigma(X, W), \varphi Z)=g\left(\widetilde{\nabla}_{W} X, \varphi Z\right)=-g\left(\varphi \widetilde{\nabla}_{W} X, Z\right) .
$$

Using a covariant derivative property of $\varphi$ and (2.2), we find

$$
g\left(A_{\varphi Z} X, W\right)=-g\left(\widetilde{\nabla}_{W} \varphi X, Z\right) .
$$


Then from (2.3) and (1.1), we get $g\left(A_{\varphi Z} X, W\right)=0$, i.e., $A_{\varphi Z} X$ has no components in $T M_{\perp}$. Therefore, from Lemma 3.1, the relation (3.4) holds.

Conversely, if $M$ is a contact CR-submanifold with the invariant and anti-invariant distributions $\mathcal{D}$ and $\mathcal{D}^{\perp} \oplus\langle\xi\rangle$ such that the given condition (3.4) holds, then, by Lemma 2.1, $\mathcal{D}^{\perp} \oplus\langle\xi\rangle$ is always integrable and for any $X \in \mathcal{D}$ and $Z, W \in \mathcal{D}^{\perp} \oplus\langle\xi\rangle$, we have

$$
g\left(\nabla_{Z} W, \varphi X\right)=g\left(\left(\widetilde{\nabla}_{Z} \varphi\right) W, X\right)-g\left(\widetilde{\nabla}_{Z} \varphi W, X\right) .
$$

Using (2.2) and (2.3), we get

$$
g\left(\nabla_{Z} W, \varphi X\right)=g\left(\varphi W, \widetilde{\nabla}_{Z} X\right)=g(\sigma(X, Z), \varphi W)=g\left(A_{\varphi W} X, Z\right) .
$$

Then from (3.4), we get $g\left(\nabla_{Z} W, \varphi X\right)=0$, which means that the leaves of the distribution $\mathcal{D}^{\perp} \oplus\langle\xi\rangle$ are totally geodesic in $M$. On the other hand, on a contact CR-submanifold from Lemma 2.3, we have

$$
g([X, Y], Z)=g\left(A_{\varphi Z} X, \varphi Y\right)-g\left(A_{\varphi Z} Y, \varphi X\right),
$$

for any $X, Y \in \mathcal{D}$ and $Z \in \mathcal{D}^{\perp} \oplus\langle\xi\rangle$. Using (3.4), (2.1) and the fact that the structure vector field $\xi$ is orthogonal to $\mathcal{D}$, we get $g([X, Y], Z)=0$, which means that $\mathcal{D}$ is integrable. Let us consider a leaf $M_{T}$ of $\mathcal{D}$ in $M$ and let $\sigma^{\#}$ be the second fundamental from of $M_{T}$ in $M$, then we have

$$
g\left(\sigma^{\#}(X, Y), Z\right)=g\left(\nabla_{Y} X, Z\right)=g\left(\varphi \widetilde{\nabla}_{Y} X, \varphi Z\right)+\eta(Z) g\left(\widetilde{\nabla}_{Y} X, \xi\right),
$$

which on using the covariant derivative property of $\varphi$ and the orthogonality of vector fields, we have

$$
g\left(\sigma^{\#}(X, Y), Z\right)=g\left(\widetilde{\nabla}_{Y} \varphi X, \varphi Z\right)-g\left(\left(\widetilde{\nabla}_{Y} \varphi\right) X, \varphi Z\right)-\eta(Z) g\left(\widetilde{\nabla}_{Y} \xi, X\right) .
$$

Using (2.2), we get

$$
g\left(\sigma^{\#}(X, Y), Z\right)=-g\left(\varphi X, \widetilde{\nabla}_{Y} \varphi Z\right)-\eta(Z) g(X, Y) .
$$

Then from (2.3), we obtain

$$
g\left(\sigma^{\#}(X, Y), Z\right)=g\left(\varphi X, A_{\varphi Z} Y\right)-\eta(Z) g(X, Y) .
$$

From (3.4), we find

$$
g\left(\sigma^{\#}(X, Y), Z\right)=-(Z \mu) g(X, Y)
$$

or equivalently

$$
\sigma^{\#}(X, Y)=-\vec{\nabla} \mu g(X, Y)
$$

where $\vec{\nabla} \mu$ is gradient of the function $\mu$, which means that $M_{\mathrm{T}}$ is totally umbilical in $M$ with the mean curvature $\mathrm{H}^{\#}=-\vec{\nabla} \mu$. Also, it is easy to prove that $\mathrm{H}^{\#}$ is parallel corresponding to the normal connection $\mathrm{D}^{\#}$ of $M_{\mathrm{T}}$ in $M$ (see [16]). Thus, $M_{\mathrm{T}}$ is an extrinsic sphere in $M$. Hence, by a result of Hiepko [9] we conclude that $M$ is a warped product submanifold, which proves the theorem completely.

Now, we set the following orthonormal frame for the warped product submanifold $M=M_{\perp} \times_{f} M_{T}$ of a $(2 n+1)$-dimensional Kenmotsu manifold $\widetilde{M}$ with the fiber $M_{T}$ of dimension $2 p$ and the base $M_{\perp}$ of dimension $q+1$ such that $\xi$ is tangent to $M_{\perp}$. Let us consider the tangent spaces of $M_{T}$ and $M_{\perp}$ by $\mathcal{D}$ and $\mathcal{D}^{\perp} \oplus\langle\xi\rangle$, respectively. We set the orthonormal frame fields of $\mathcal{D}$ and $\mathcal{D}^{\perp} \oplus\langle\xi\rangle$, respectively as $\left\{e_{1}, \cdots, e_{p}, e_{p+1}=\varphi e_{1}, \cdots, e_{2 p}=\varphi e_{p}\right\}$ and $\left\{e_{2 p+1}=e_{1}^{*}, \cdots, e_{2 p+q}=e_{q}^{*}, e_{2 p+q+1}=e_{q+1}^{*}=\xi\right\}$. 
Then the orthonormal frame fields of the normal subbundles of $\varphi \mathcal{D}^{\perp}$ and $\nu$, respectively are $\left\{\boldsymbol{e}_{\mathrm{m}+1}=\right.$ $\left.\varphi e_{1}^{*}, \cdots, e_{m+q}=\varphi e_{q}^{*}\right\}$ and $\left\{e_{m+q+1}, \cdots, e_{2 n+1}\right\}$.

A warped product submanifold $M=M_{1} \times_{f} M_{2}$ of a Riemannian manifold $\widetilde{M}$ is said to be mixed totally geodesic, if $\sigma(X, Z)=0$, for any $X \in T M_{1}$ and $Z \in T M_{2}$, where $M_{1}$ and $M_{2}$ are any Riemannian submanifolds of $\widetilde{M}$.

Now, we establish the following inequality for the squared norm of the second fundamental form of $M=M_{\perp} \times_{f} M_{T}$ of a Kenmotsu manifold $\widetilde{M}$.

Theorem 3.4. Let $\mathrm{M}=\mathrm{M}_{\perp} \times_{\mathrm{f}} \mathrm{M}_{\mathrm{T}}$ be a warped product submanifold of a Kenmotsu manifold $\widetilde{M}$ such that $\xi \in \mathrm{TM}_{\perp}$, where $\mathrm{M}_{\perp}$ is an anti-invariant submanifold and $\mathrm{M}_{\mathrm{T}}$ is an invariant submanifold of $\widetilde{M}$. Then:

(i) The squared norm of the second fundamental form $\sigma$ of $M$ satisfies

$$
\|\sigma\|^{2} \geqslant 2 p\left[\|\vec{\nabla} \ln f\|^{2}-1\right],
$$

where $2 p=\operatorname{dim} M_{\mathrm{T}}$ and $\vec{\nabla} \ln \mathrm{f}$ is gradient of the function $\ln \mathrm{f}$ along $\mathrm{M}_{\perp}$.

(ii) If equality sign in (i) holds identically, then $\mathrm{M}_{\perp}$ and $\mathrm{M}_{\mathrm{T}}$ are totally geodesic and totally umbilical submanifolds of $\widetilde{M}$, respectively. Moreover, $M$ is a mixed totally geodesic submanifold of $\widetilde{M}$.

Proof. By definition of $\sigma$, we have

$$
\|\sigma\|^{2}=\sum_{i, j=1}^{m} g\left(\sigma\left(e_{i}, e_{j}\right), \sigma\left(e_{i}, e_{j}\right)\right)=\sum_{r=m+1}^{2 n+1} \sum_{i, j=1}^{m} g\left(\sigma\left(e_{i}, e_{j}\right), e_{r}\right)^{2} .
$$

Then by using the above mentioned frame, we derive

$$
\begin{aligned}
\|\sigma\|^{2}= & \sum_{r=m+1}^{2 n+1} \sum_{i, j=1}^{2 p} g\left(\sigma\left(e_{i}, e_{j}\right), e_{r}\right)^{2}+2 \sum_{r=m+1}^{2 n+1} \sum_{i, j=1}^{2 p} \sum_{j=1}^{q+1} g\left(\sigma\left(e_{i}, e_{j}^{*}\right), e_{r}\right)^{2} \\
& +\sum_{r=m+1}^{2 n+1} \sum_{i, j=1}^{q+1} g\left(\sigma\left(e_{i}^{*}, e_{j}^{*}\right), e_{r}\right)^{2} .
\end{aligned}
$$

By leaving second and third positive terms in the right hand side of (3.5), the above expression will be

$$
\|\sigma\|^{2} \geqslant \sum_{r=1}^{q} \sum_{i, j=1}^{2 p} g\left(\sigma\left(e_{i}, e_{j}\right), \varphi e_{r}^{*}\right)^{2}+\sum_{r=m+q+1}^{2 n+1} \sum_{i, j=1}^{2 p} g\left(\sigma\left(e_{i}, e_{j}\right), e_{r}\right)^{2} .
$$

The second term in the right hand side of the above relation has the $v$-components only, therefore we will also leave this term and thus for the frame of $\mathcal{D}$, the inequality (3.6) reduces to

$$
\begin{aligned}
\|\sigma\|^{2} \geqslant & \sum_{r=1}^{q} \sum_{i, j=1}^{p} g\left(\sigma\left(e_{i}, \varphi e_{j}\right), \varphi e_{r}^{*}\right)^{2}+\sum_{r=1}^{q} \sum_{i, j=1}^{p} g\left(\sigma\left(\varphi e_{i}, e_{j}\right), \varphi e_{r}^{*}\right)^{2} \\
& +\sum_{r=1}^{q} \sum_{i, j=1}^{p} g\left(\sigma\left(\varphi e_{i}, \varphi e_{j}\right), \varphi e_{r}^{*}\right)^{2}+\sum_{r=1}^{q} \sum_{i, j=1}^{p} g\left(\sigma\left(e_{i}, e_{j}\right), \varphi e_{r}^{*}\right)^{2} .
\end{aligned}
$$

Using Lemma 3.1 (i) and the relations (3.1), (3.2), (3.3), the third and the last terms of right hand side are identically zero. Thus, we derive

$$
\|\sigma\|^{2} \geqslant p \sum_{r=1}^{q}\left[\eta\left(e_{r}^{*}\right)-e_{r}^{*} \ln f\right]^{2}+p \sum_{r=1}^{q}\left[e_{r}^{*} \ln f-\eta\left(e_{r}^{*}\right)\right]^{2}
$$




$$
=2 p \sum_{r=1}^{q+1}\left[\eta\left(e_{r}^{*}\right)-e_{r}^{*} \ln f\right]^{2}-2 p\left[\eta\left(e_{q+1}^{*}\right)-\left(e_{q+1}^{*} \ln f\right)\right]^{2} .
$$

Since $e_{\mathbf{q}+1}^{*}=\xi$ and for a warped product Riemannian submanifold $M$ of a Kenmotsu manifold $\widetilde{M}$, $(\xi \ln f)=1[1,17]$. Then the above inequality will be

$$
\begin{aligned}
\|\sigma\|^{2} & \geqslant 2 p\left[\eta\left(e_{q+1}^{*}\right)^{2}+\sum_{r=1}^{q+1}\left(e_{r}^{*} \ln f\right)^{2}-2 \sum_{r=1}^{q+1}\left(e_{r}^{*} \ln f\right) \eta\left(e_{r}^{*}\right)\right] \\
& =2 p\left[\|\vec{\nabla} \ln f\|^{2}-1\right]
\end{aligned}
$$

which is inequality (i). If the equality holds in (i), then from the remaining terms in (3.5), we get

$$
\sigma\left(\mathcal{D}^{\perp}, \mathcal{D}\right)=0, \quad \sigma\left(\mathcal{D}^{\perp}, \mathcal{D}^{\perp}\right)=0 .
$$

Also, from the remaining second term in the right hand side of (3.6), we find

$$
\sigma(\mathcal{D}, \mathcal{D}) \perp v \Rightarrow \sigma(\mathcal{D}, \mathcal{D}) \in \varphi \mathcal{D}^{\perp} .
$$

The second condition of (3.7) implies that $M_{\perp}$ is a totally geodesic submanifold of $\widetilde{M}$ due to $M_{\perp}$ being totally geodesic in $M[4,5]$. On the other hand, (3.8) implies that $M_{T}$ is totally umbilical in $\widetilde{M}$ with the fact that $M_{\mathrm{T}}$ is totally umbilical in $M[4,5]$. Moreover, all conditions of (3.7) and (3.8) imply that $M$ is a mixed totally geodesic submanifold of $\widetilde{M}$. Hence the proof is complete.

In [14], Olteanu established the following estimation for the squared norm of the second fundamental form for contact CR-doubly warped products in Kenmotsu manifolds.

Theorem 3.5 ([14]). Let $\widetilde{M}$ be a $(2 \mathrm{~m}+1)$-dimensional Kenmotsu manifold and $M=f_{2} M_{1} \times_{f_{1}} M_{2}$ an $n$ dimensional contact $C R$-doubly warped product submanifold, such that $M_{1}$ is a $(2 \alpha+1)$-dimensional invariant submanifold tangent to $\xi$ and $M_{2}$ is a $\beta$-dimensional anti-invariant submanifold of $\widetilde{M}$. Then:

(i) The squared norm of the second fundamental form of $M$ satisfies

$$
\|\sigma\|^{2} \geqslant 2 \beta\left(\left\|\vec{\nabla}\left(\ln f_{1}\right)\right\|^{2}-1\right),
$$

where $\vec{\nabla}\left(\ln \mathrm{f}_{1}\right)$ is the gradient of $\ln \mathrm{f}_{1}$.

(ii) If the equality sign of (3.9) holds identically, then $M_{1}$ is a totally geodesic submanifold and $M_{2}$ is a totally umbilical submanifold of $M$. Moreover, $M$ is a minimal submanifold of $\widetilde{M}$.

Now, we give the following examples of non-trivial warped product contact CR-submanifolds of the forms $M_{\perp} \times_{f} M_{T}$ and $M_{\perp} \times_{f} M_{T}$ and in both the cases the structure vector field $\xi$ is tangent to the base manifold.

Example 3.6. Consider the Kenmotsu manifold $\widetilde{M}=\mathbb{R} \times_{f} \mathbb{C}^{4}$ with the structure $(\varphi, \xi, \eta, g)$ is given by

$$
\begin{gathered}
\varphi\left\{\sum_{i=1}^{4}\left(X_{i} \frac{\partial}{\partial x^{i}}+Y_{i} \frac{\partial}{\partial y^{i}}\right)+Z \frac{\partial}{\partial t}\right\}=\sum_{i=1}^{4}\left(-Y_{i} \frac{\partial}{\partial x^{i}}+X_{i} \frac{\partial}{\partial y^{i}}\right), \\
\xi=2 e^{-t}\left(\frac{\partial}{\partial t}\right), \quad \eta=\frac{1}{2} e^{t} d t, \quad \text { and } g=\eta \otimes \eta+\frac{e^{2 t}}{4} \sum_{i=1}^{4}\left(d x^{i} \otimes d x^{i}+d y^{i} \otimes d y^{i}\right) .
\end{gathered}
$$


Consider the submanifold $M$ of $\widetilde{M}$ defined by

$$
x(u, v, w, s, t)=2\left(e^{-t} u, 0, w, 0,0, e^{-t} v, s, 0, t\right) .
$$

Then $M$ is a contact CR-warped product submanifold with the integrable invariant and anti-invariant distributions $\mathcal{D}=\left\{e_{3}, e_{4},\right\}$ and $\mathcal{D}^{\perp}=\left\{e_{1}, e_{2}, e_{5}\right\}$ such that

$$
\begin{gathered}
e_{1}=\frac{2}{e^{t}}\left(\frac{\partial}{\partial x^{1}}\right), e_{2}=\frac{2}{e^{t}}\left(\frac{\partial}{\partial y^{2}}\right), e_{3}=2\left(\frac{\partial}{\partial x^{3}}\right), \\
e_{4}=2\left(\frac{\partial}{\partial y^{3}}\right), \quad e_{5}=2\left(\frac{\partial}{\partial t}\right)=e^{t} \xi .
\end{gathered}
$$

Consider, the integral manifolds corresponding to the distributions $\mathcal{D}$ and $\mathcal{D}^{\perp}$ by $M_{T}$ and $M_{\perp}$, respectively. Then their corresponding Riemannian metrics are respectively given by $g_{M_{T}}=e^{2 t}\left((d w)^{2}+(d s)^{2}\right)$ and $g_{M_{\perp}}=d t^{2}+d u^{2}+d v^{2}$. Thus $M=M_{\perp} \times_{f} M_{T}$ is a warped product submanifold isometrically immersed in $\widetilde{M}$ with metric $g=g_{M_{\perp}}+e^{2 t} g_{M_{T}}$ and warping function $f=e^{t}$.

Example 3.7. Consider a submanifold of $\mathbb{R}^{7}$ with the Cartesian coordinates $\left(x_{1}, y_{1}, x_{2}, y_{2}, x_{3}, y_{3}, t\right)$ and the almost contact structure

$$
\varphi\left(\frac{\partial}{\partial x_{i}}\right)=\frac{\partial}{\partial y_{i}}, \quad \varphi\left(\frac{\partial}{\partial y_{j}}\right)=-\frac{\partial}{\partial x_{j}}, \quad \varphi\left(\frac{\partial}{\partial t}\right)=0, \quad 1 \leqslant i, j \leqslant 3 .
$$

Then for some smooth functions $\lambda_{i}, v_{j}$ and $\mu$ on $\mathbb{R}^{7}$, for any $i, j=1,2,3$, consider a vector field $X=$ $\lambda_{i} \frac{\partial}{\partial x_{i}}+v_{j} \frac{\partial}{\partial y_{j}}+\mu \frac{\partial}{\partial t} \in \mathbb{R}^{7}$, we have

$$
g(X, X)=\lambda_{i}^{2}+v_{j}^{2}+\mu^{2}, \quad g(\varphi X, \varphi X)=\lambda_{i}^{2}+v_{j}^{2}
$$

and

$$
\varphi^{2}(X)=-\lambda_{i} \frac{\partial}{\partial x_{i}}-v_{j} \frac{\partial}{\partial y_{j}}=-X+\eta(X) \xi,
$$

for any $i, j=1,2,3$. It is clear that $g(\varphi X, \varphi X)=g(X, X)-\eta^{2}(X)$. Thus, $(\varphi, \xi, \eta, g)$ is an almost contact metric structure on $\mathbb{R}^{7}$. Let us consider a submanifold $M$ of $\mathbb{R}^{7}$ defined by the immersion $\chi$ as follows

$$
\chi(u, v, w, t)=(u \cos w, v \cos w, u \sin w, v \sin w, 0,0, t) .
$$

Then the tangent bundle TM is spanned by the following orthogonal vector fields

$$
\begin{gathered}
e_{1}=\cos w \frac{\partial}{\partial x_{1}}+\sin w \frac{\partial}{\partial x_{2}}, \quad e_{2}=\cos w \frac{\partial}{\partial y_{1}}+\sin w \frac{\partial}{\partial y_{2}}, \\
e_{3}=-u \sin w \frac{\partial}{\partial x_{1}}-v \sin w \frac{\partial}{\partial y_{1}}+u \cos w \frac{\partial}{\partial x_{2}}+v \cos w \frac{\partial}{\partial y_{2}}, \quad e_{4}=\frac{\partial}{\partial t} .
\end{gathered}
$$

Then, we find

$$
\begin{gathered}
\varphi e_{1}=\cos w \frac{\partial}{\partial y_{1}}+\sin w \frac{\partial}{\partial y_{2}}, \quad \varphi e_{2}=-\cos w \frac{\partial}{\partial x_{1}}-\sin w \frac{\partial}{\partial x_{2}} \\
\varphi e_{3}=-u \sin w \frac{\partial}{\partial y_{1}}+v \sin w \frac{\partial}{\partial x_{1}}+u \cos w \frac{\partial}{\partial y_{2}}-v \cos w \frac{\partial}{\partial x_{2}}, \quad \varphi e_{4}=0 .
\end{gathered}
$$


Thus $M$ is a contact $C R$-submanifold with invariant distribution $\mathcal{D}=\operatorname{span}\left\{e_{1}, e_{2}\right\}$ and anti-invariant distribution $\mathcal{D}^{\perp}=\operatorname{span}\left\{e_{3}\right\}$ such that $\xi=e_{4}$ tangent to $\mathcal{D}$. Also, it is easy to see that both the distributions $\mathcal{D} \oplus\left\langle\xi>\right.$ and $\mathcal{D}$ are integrable. If we denote the integral manifolds of $\mathcal{D}$ and $\mathcal{D}^{\perp} \oplus\left\langle\xi>\right.$ by $M_{\mathrm{T}}$ and $M_{\perp}$ respectively, then the metric tensor $g_{M}$ of $M$ is given by

$$
g_{M}=d u^{2}+d v^{2}+d t^{2}+\left(u^{2}+v^{2}\right) d w^{2},
$$

where $g_{M_{T}}=d u^{2}+d v^{2}+d t^{2}$ is the metric tensor of $M_{T}$ and $g_{M_{\perp}}=\left(u^{2}+v^{2}\right) d w^{2}$ is the metric tensor of $M_{\perp}$. Thus $M$ is a warped product contact CR-submanifold $M=M_{T} \times_{f} M_{\perp}$ with the warping function $f=\sqrt{u^{2}+v^{2}}$.

\section{References}

[1] K. Arslan, R. Ezentas, I. Mihai, C. Murathan, Contact CR-warped product submanifolds in Kenmotsu space forms, J. Korean Math. Soc., 42 (2005), 1101-1110. 1, 3, 3

[2] M. Atçeken, Warped product semi-slant submanifolds in Kenmotsu manifolds, Turkish J. Math., 34 (2010), 425-432.

[3] M. Atçeken, Contact CR-warped product submanifolds in Kenmotsu space forms, Bull. Iranian Math. Soc., 39 (2013), 415-429. 1

[4] R. L. Bishop, B. O’Neill, Manifolds of negative curvature, Trans. Amer. Math. Soc., 145 (1969), 1-49. 1, 1, 3

[5] B.-Y. Chen, Geometry of warped product CR-submanifolds in Kaehler manifolds, Monatsh. Math., 133 (2001), $177-195$. 1,3

[6] B.-Y. Chen, Pseudo-Riemannian geometry, -invariants and applications, With a foreword by Leopold Verstraelen, World Scientific Publishing Co. Pte. Ltd., Hackensack, NJ, (2011). 1

[7] B.-Y. Chen, Geometry of warped product submanifolds: a survey, J. Adv. Math. Stud., 6 (2013), 1-43. 1

[8] I. Hasegawa, I. Mihai, Contact CR-warped product submanifolds in Sasakian manifolds, Geom. Dedicata, 102 (2003), 143-150. 1

[9] S. Hiepko, Eine innere Kennzeichnung der verzerrten Produkte, (German) Math. Ann., 241 (1979), 209-215. 3

[10] K. Kenmotsu, A class of almost contact Riemannian manifolds, Tôhoku Math. J., 24 (1972), 93-103. 2

[11] V. A. Khan, K. A. Khan, S. Uddin, A note on warped product submanifolds of Kenmotsu manifolds, Math. Slovaca, 61 (2011), 79-92. 1

[12] V. A. Khan, M. Shuaib, Some warped product submanifolds of a Kenmotsu manifold, Bull. Korean Math. Soc., 51 (2014), 863-881. 2.1

[13] C. Murathan, K. Arslan, R. Ezentas, I. Mihai, Warped product submanifolds in Kenmotsu space forms, Taiwanese J. Math., 10 (2006), 1431-1441. 1

[14] A. Olteanu, Contact CR-doubly warped product submanifolds in Kenmotsu space forms, JIPAM. J. Inequal. Pure Appl. Math., 10 (2009), 7 pages. 1, 3, 3.5

[15] S. Tanno, The automorphism groups of almost contact Riemannian manifolds, Tôhoku Math. J., 21 (1969), 21-38. 2

[16] S. Uddin, F. R. Al-Solamy, Warped product pseudo-slant submanifolds of cosymplectic manifolds, Ann. St. Univ. Iasi, (In press). 3

[17] S. Uddin, V. A. Khan, K. A. Khan, Warped product submanifolds of a Kenmotsu manifold, Turkish J. Math., 36 (2012), 319-330. 1, 3

[18] S. Uddin, C. Ozel, V. A. Khan, Warped product CR-submanifolds of Lorentzian $\beta$-Kenmotsu manifolds, Publ. Inst. Math. (Beograd) (N.S.), 92 (2012), 157-163. 1 